\title{
OBSERVER-BASED INPUT-OUTPUT LINEARIZATION CONTROL OF A MULTIVARIABLE CONTINUOUS CHEMICAL REACTOR
}

\author{
Bouhamida Mohamed, Daaou Bachir, Mansouri Abdellah, \\ and Chenafa Mohammed
}

\begin{abstract}
The goal of this paper is to develop a nonlinear observerbased control strategy for a multi-variables continuous stirred tank reactor (CSTR). A new robust nonlinear observer is constructed to estimate the whole process state variables. The observer is coupled with a nonlinear controller, designed based on the input-output linearization for controlling the concentration and reactor temperature. The closed loop system is shown to be globally asymptotically stable based on Lyapunov arguments. Finally, computer simulations are developed for showing the performance of the proposed controller.
\end{abstract}

\section{Introduction}

Continuous stirred tank reactors (CSTR) are ones of the most important plants in chemical industry. In practice, however, the control of these reactors poses a number of challenging problems. These problems arise from the presence of severe nonlinearities of these chemical reactors, as well as for monitoring and control applications, only a few measurements are available, either because the measuring devices do not exist or are too expensive. Therefore, we can deduce that the main difficulties arising in the control of CSTR's reactors arrive from two main sources: the process complexity and the difficulty to have reliable measurements of state variables ([1], [16]). In recent years, a various design methods of nonlinear control strategies have been proposed. Most of these based on differential geometric concepts ([15]-[18], [23]). This method allows a certain class of systems to be linearized using state feedback and coordinate transformations ([12], [14]). Extensions of the method like adaptive linearization ([21], [25]), robust linearization [24], and asymptotically exact linearization [13] account for small model-plant-mismatch.

Received December 22, 2010

2010 Mathematics Subject Classification. 93C83.

Key words and phrases. non-linear observer, feedback linéarizing controller, global stability, chemical reactor. 
Concerning the state estimation, there are many works dealing with the observation techniques to estimate the state variables in chemical reactors ([4][11], [26]).

The main purpose of this work is to develop an observer-based nonlinear control scheme for a multi-input multi-output continuous chemical reactor (CSTR). We apply a robust non-linear observer, as proposed by Daaou et al. [7] in order to estimate the concentrations. The controller is constructed through feedback linearization. Keeping in mind that no separation principle exists for nonlinear systems, the study of the asymptotical stability of the closed-loop system including the observer dynamics has been established, which is the main contribution of this work.

This article is arranged as follows. In Section 2, the reactor model is presented. In Section 3, a non-linear observer for estimating concentrations is constructed. Section 4 is devoted to closed loop control, where we use Lyapunov arguments to prove the closed loop stability. In Section 5, the computer simulations were developed to illustrate the effectiveness of the proposed approach. Finally, we will close the paper with some concluding remarks.

\section{System description}

The continuous stirred chemical reactor (CSTR) has been perhaps one of the most widely studied unit operation, from both dynamic analysis and control perspectives. The typical diagram of this reactor is shown in Figure 1 in which the series reactions, $A \stackrel{k_{1}}{\rightarrow} B \stackrel{k_{2}}{\rightarrow} C$, take place in the liquid phase.

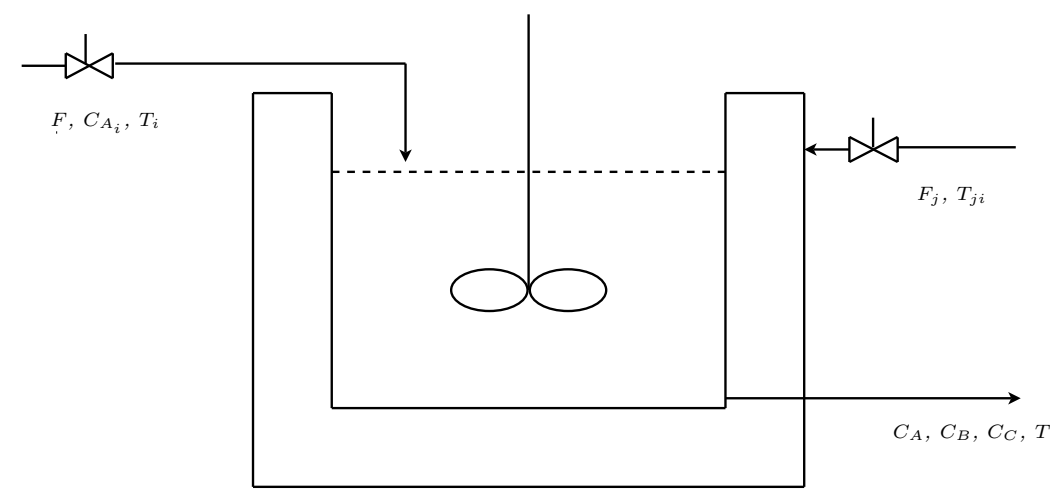

FiguRE 1. Schematic of the multivariable non-isothermal chemical reactor.

Here $C_{A}, C_{B}$ and $C_{C}$ are the outlet concentrations of the reactant $A, B$ and $C$ respectively, $C_{A i}$ inlet concentration of the reactant $A, T$ reactor outlet temperature, $T_{i}$ reactor inlet temperature, $T_{j}$ jacket temperature, $T_{j i}$ jacket inlet temperature, $F$ feed flow rate to reactor. 
TABLE 1. Parameter values of the reactor.

\begin{tabular}{|l|l|l|}
\hline Parameter & value & Unit \\
\hline$\alpha$ & -0.1914 & - \\
$\beta_{1}$ & 0.0072 & - \\
$\beta_{2}$ & 0.0120 & - \\
$k_{01}$ & $15.155 \times 10^{8}$ & $\mathrm{~min}^{-1}$ \\
$E_{1}$ & 60 & JJmol $^{-1}$ \\
$k_{02}$ & $38.22 \times 10^{6}$ & $\mathrm{~min}^{-1}$ \\
$E_{2}$ & 55 & JJmol $^{-1}$ \\
$V$ & 0.009 & $\mathrm{~m}^{3}$ \\
$T_{i}$ & 293 & $\mathrm{~K}$ \\
$T_{i j}$ & 343 & $\mathrm{~K}$ \\
$C_{A i}$ & 100 & $\mathrm{~mol}^{-3}$ \\
$R$ & 08.314 & J.mol \\
\hline
\end{tabular}

In this paper some assumptions have been introduced in the attempt to reduce the model complexity. In particular, we will assume the following:

(i) There is a complete uniformity of concentration and temperature within the reactor and a complete uniformity of temperature inside the jacket.

(ii) The reactor and jacket volumes are constant.

(iii) The thermal exchange between the reactor and the jacket is expressed by a constant global heat exchange coefficient $U$ and the amount of heat retained in the reactor walls is negligible.

(iv) The reaction enthalpies $\Delta H_{i}$ are independent of temperature.

(v) The heat capacities of the process fluids are constant.

Under the above assumptions, the material and energy balances applied to the jacket reactor give the mathematical model [7].

(1) $\dot{T}=-\frac{U A}{\rho C_{p} V}\left(T-T_{j}\right)+\frac{F}{V}\left(T_{i}-T\right)-\frac{\Delta H_{1}}{\rho C_{p}} K_{1}(T) C_{A}-\frac{\Delta H_{2}}{\rho C_{p}} K_{2}(T) C_{B}$,

(2) $\dot{C}_{A}=-K_{1}(T) C_{A}+\frac{F}{V}\left(C_{A i}-C_{A}\right)$,

(3) $\dot{C}_{B}=K_{1}(T) C_{A}-K_{2}(T) C_{B}-\frac{F}{V} C_{B}$,

(4) $\dot{C}_{C}=K_{2}(T) C_{B}-\frac{F}{V} C_{C}$,

(5) $y_{1}=T$,

(6) $y_{2}=C_{C}$,

with $K_{i}(T)=k_{i 0} \exp \frac{-E_{i}}{R T}, i=1,2$, where $U$ is the overall heat-transfer coefficient, $A$ heat transfer surface area, $C_{p}$ heat capacity of feed and product, $C_{p j}$ heat capacity of jacket fluid, $E_{i}(\mathrm{i}=1,2)$ activation energy, $R$ universal gas constant, $k_{0 i}(i=1,2)$ pre-exponential factor, $\Delta H_{i}(i=1,2)$ heat of reaction $\rho$ density of mixture in reactor and $\rho_{j}$ density of jacket fluid. 
The model (1-6) has the following form:

$$
\begin{aligned}
& \dot{x}=f(x)+g(x) u \\
& y=C_{x} x,
\end{aligned}
$$

where $x=\left[T, C_{A}, C_{B}, C_{C}\right]^{T}, u=\left[F_{0}, T_{j}\right]^{T}$ and

$$
\begin{gathered}
f(x)=\left[\begin{array}{c}
\alpha x_{1}+\beta_{1} K_{1}\left(x_{1}\right) x_{2}+\beta_{2} K_{2}\left(x_{1}\right) x_{3} \\
-K_{1}\left(x_{1}\right) x_{2} \\
K_{1}\left(x_{1}\right) x_{2}-K_{2}\left(x_{1}\right) x_{3} \\
K_{2}\left(x_{1}\right) x_{3}
\end{array}\right], g(x)=\left[\begin{array}{cc}
\frac{1}{V}\left(T_{i}-x_{1}\right) & -\alpha \\
\frac{1}{V}\left(C_{A i}-x_{2}\right) & 0 \\
-\frac{1}{V} x_{3} & 0 \\
-\frac{1}{V} x_{4} & 0
\end{array}\right], \\
C_{x}=\left[\begin{array}{llll}
1 & 0 & 0 & 0 \\
0 & 0 & 0 & 1
\end{array}\right] \text { with } \alpha_{1}=-\frac{U A}{\rho C_{p} V}, \beta_{1}=-\frac{\Delta H_{1}}{\rho C_{p}}, \beta_{2}=-\frac{\Delta H_{2}}{\rho C_{p}} .
\end{gathered}
$$

The reactor parameter values are given in (1) [7].

\section{Nonlinear observer design}

$$
\begin{aligned}
& \dot{x}=\underbrace{\left[\begin{array}{c}
\alpha x_{1}+\beta_{1} K_{1}\left(x_{1}\right) x_{2}+\beta_{2} K_{2}\left(x_{1}\right) x_{3} \\
-K_{1}\left(x_{1}\right) x_{2} \\
K_{1}\left(x_{1}\right) x_{2}-K_{2}\left(x_{1}\right) x_{3} \\
K_{2}\left(x_{1}\right) x_{3}
\end{array}\right]}_{f(x)}+\underbrace{\left[\begin{array}{cc}
\frac{1}{V}\left(T_{i}-x_{1}\right) & \alpha_{1} \\
\frac{1}{V}\left(C_{A i}-x_{3}\right) & 0 \\
-\frac{1}{V} x_{3} & 0 \\
-\frac{1}{V} x_{4} & 0
\end{array}\right]}_{g(x)}\left[\begin{array}{l}
u_{1} \\
u_{2}
\end{array}\right] \\
& y=\left[\begin{array}{l}
h_{1}(x) \\
h_{2}(x)
\end{array}\right]=\left[\begin{array}{l}
x_{1} \\
x_{4}
\end{array}\right] .
\end{aligned}
$$

By using the usual variable change:

$$
x \rightarrow \Psi(x)=\left[\begin{array}{c}
h(x) \\
L_{f}[h(x)]
\end{array}\right]=\left[\begin{array}{c}
x_{1} \\
x_{4} \\
L_{f}\left[x_{1}\right] \\
L_{f}\left[x_{4}\right]
\end{array}\right]=\left[\begin{array}{c}
z_{1} \\
z_{2}
\end{array}\right]
$$

with $z_{1}=\left[x_{1}, x_{4}\right]^{T}, z_{2}=\left[L_{f}\left[x_{1}\right], L_{f}\left[x_{4}\right]\right]^{T}, L_{f}(h(x))=\sum \frac{\partial h}{\partial x_{i}} f_{i}$.

The change of coordinates gives the following system:

$$
\begin{gathered}
\dot{z}=\left[\begin{array}{c}
\dot{z_{1}} \\
\dot{z_{2}}
\end{array}\right]=\underbrace{\left[\begin{array}{ll}
0 & 1 \\
0 & 0
\end{array}\right]}_{A} \underbrace{\left[\begin{array}{l}
z_{1} \\
z_{2}
\end{array}\right]}_{z}+\underbrace{\left[\begin{array}{c}
0 \\
\varphi(z)
\end{array}\right]}_{\Phi(z)}+\underbrace{\left[\begin{array}{c}
B_{1}(z, u) \\
B_{2}(z, u)
\end{array}\right]}_{B(z, u)} \\
y=z_{1}, \\
\text { where } \varphi(z)=\left[\begin{array}{l}
L_{f}^{2}\left[x_{1}\right] \\
L_{f}^{2}\left[x_{4}\right]
\end{array}\right], B_{1}(z, u)=\left[\begin{array}{l}
L_{g}\left[x_{1}\right] \\
L_{g}\left[x_{4}\right]
\end{array}\right], B_{2}(z, u)=\left[\begin{array}{l}
L_{f} L_{g}\left[x_{1}\right] \\
L_{f} L_{g}\left[x_{4}\right]
\end{array}\right] .
\end{gathered}
$$

We now state the following assumptions:

Assumption 1. The function $\varphi(z)$ is globally Lipschitz with respect to $z$.

Assumption 2. The functions $B_{1}(z, u)$ and $B_{2}(z, u)$ are globally Lipschitz with respect to $z$ uniformly with respect to the inputs $u$. 
Theorem 1. Under the above assumptions, the nonlinear observer given by:

$$
\dot{\hat{z}}=A \hat{z}+\Phi(\hat{z})+B(\hat{z}, u)-G_{\theta}(C \hat{z}-y)
$$

is an exponential observer for the system (10) for $\theta$ sufficiently large, with

$$
\begin{aligned}
A & =\left[\begin{array}{ll}
0 & 1 \\
0 & 0
\end{array}\right], \Phi(\hat{z})=\left[\begin{array}{c}
0 \\
\varphi(\hat{z})
\end{array}\right], B(\hat{z}, u)=\left[\begin{array}{c}
B_{1}\left(\hat{z_{1}}, u\right) \\
B_{2}\left(\hat{z_{2}}, u\right)
\end{array}\right], C=[1,0], \\
G_{\theta} & =S_{\theta}^{-1} G=\left[\begin{array}{c}
\theta G_{1} \\
\theta^{2} G_{2}
\end{array}\right], \theta>0,
\end{aligned}
$$

where $G$ is a constant matrix so that $(A-G C)$ is stable and $S_{\theta}$ is the solution of the Lyapunov equation:

$$
\dot{S}_{\theta}=-\theta S_{\theta}+A^{T} S_{\theta}-S_{\theta} A+C_{z} C_{z}^{T}=0 .
$$

When the inverse variable change $\Psi^{-1}$ is applied, the equation of the observer in the original $x$-coordinate system $\hat{x}=\Psi^{-1}(\hat{z})$ is given by:

$$
\begin{aligned}
& \dot{\hat{x}}=f(\hat{x})+g(\hat{x}) u-\left[\frac{\partial \Psi(x)}{\partial x}\right]^{-1} G_{\theta}(C \hat{x}-y) \\
& \hat{y}=C_{x} \hat{x}
\end{aligned}
$$

Proof. See Appendix A.

\section{Input-output feedback linearization controller design}

In this work we consider a multiple-input/multiple-output controller whose regulated outputs are the temperature and concentration of the constituent $B$, that is, $x_{1}$ and $x_{3}$.

The linearization condition that permits to verify if a nonlinear system admits an input output linearization is the relative degree order of the system $([15],[24])$.

The relative degree of an output is the number of times that it is necessary to derive the output to reveal the input $u$.

$$
\begin{aligned}
& \dot{y}_{1}=L_{f}\left(x_{1}\right)+L_{g_{11}}\left(x_{1}\right) u_{1}+L_{g_{12}}\left(x_{1}\right) u_{2}, \\
& \dot{y}_{2}=L_{f}\left(x_{3}\right)+L_{g_{21}}\left(x_{3}\right) u_{1},
\end{aligned}
$$

with

$$
\begin{aligned}
L_{f}\left(x_{1}\right) & =\alpha x_{1}+\beta_{1} r_{1}(x)+\beta_{2} r_{2}(x), \\
L_{f}\left(x_{3}\right) & =r_{1}(x)-r_{2}(x), \\
L_{g_{11}}\left(x_{1}\right) & =\frac{1}{V}\left(T_{i}-x_{1}\right), \\
L_{g_{12}}\left(x_{1}\right) & =-\alpha, \\
L_{g_{21}}\left(x_{1}\right) & =-\frac{1}{V} x_{3},
\end{aligned}
$$

where $r_{1}(x)=K_{1}\left(x_{1}\right) x_{2}$ and $r_{2}(x)=K_{2}\left(x_{1}\right) x_{3}$. 
Then, the total degree of the system is equal to 2 .

Indeed, we have:

$$
\left[\begin{array}{l}
\dot{y}_{1} \\
\dot{y}_{2}
\end{array}\right]=\left[\begin{array}{c}
L_{f}\left(x_{1}\right) \\
L_{f}\left(x_{2}\right)
\end{array}\right]+\left[\begin{array}{cc}
L_{g_{11}}\left(x_{1}\right) & L_{g_{12}}\left(x_{1}\right) \\
L_{g_{21}}\left(x_{3}\right) & 0
\end{array}\right]\left[\begin{array}{l}
u_{1} \\
u_{2}
\end{array}\right]
$$

and we obtain the two feedbacks:

$$
\begin{aligned}
& u_{1}=\frac{v_{2}-L_{f}\left(x_{3}\right)}{L_{g_{21}}\left(x_{3}\right)}, \\
& u_{2}=\frac{v_{1}-L_{f}\left(x_{1}\right)-L_{g_{11}}\left(x_{1}\right) u_{1}}{L_{g_{12}}\left(x_{1}\right)},
\end{aligned}
$$

where $v_{1}=\hat{y}_{1}$ and $v_{2}=\hat{y}_{2}$.

It should be noted that the state variables are estimated using an observer. Then the equations (17) and (18) become:

$$
\begin{aligned}
u_{1} & =\frac{v_{2}-\hat{L}_{f}\left(x_{3}\right)}{\hat{L}_{g_{21}}\left(x_{3}\right)}, \\
u_{2} & =\frac{v_{1}-\hat{L}_{f}\left(x_{1}\right)-\hat{L}_{g_{11}}\left(x_{1}\right) u_{1}}{\hat{L}_{g_{12}}\left(x_{1}\right)}
\end{aligned}
$$

with

$$
\begin{aligned}
& v_{2}=\frac{\hat{L}_{g_{21}}\left(x_{3}\right)}{L_{g_{21}}\left(x_{3}\right)} \dot{y}_{2}-\frac{\hat{L}_{g_{21}}\left(x_{3}\right)}{L_{g_{21}}\left(x_{3}\right)} L_{f}\left(x_{3}\right)+\hat{L}_{f}\left(x_{3}\right)=\frac{\hat{x}_{3}}{x_{3}} \dot{y}_{2}-\frac{\hat{x}_{3}}{x_{3}} L_{f}\left(x_{3}\right)+\hat{L}_{f}\left(x_{3}\right), \\
& v_{1}=\dot{y}_{1}-L_{f}\left(x_{1}\right)-L_{g_{11}}\left(x_{1}\right) u_{1}+\hat{L}_{f}\left(x_{1}\right)+\hat{L}_{g_{11}}\left(x_{1}\right) u_{1},
\end{aligned}
$$

where

$$
\begin{aligned}
\hat{L}_{f}\left(x_{1}\right) & =\alpha \hat{x}_{1}+\beta_{1} r_{1}(\hat{x})+\beta_{2} r_{2}(\hat{x})+\theta \tilde{x}_{1}, \\
\hat{L}_{f}\left(x_{3}\right) & =r_{1}(\hat{x})-r_{2}(\hat{x})+\gamma_{1} \tilde{x}_{1}+\gamma_{2} \tilde{x}_{4}, \\
\hat{L}_{g_{11}}\left(x_{1}\right) & =\frac{1}{V}\left(T_{i}-\hat{x}_{1}\right), \\
\hat{L}_{g_{21}}\left(x_{3}\right) & =-\frac{1}{V} x_{3}, \\
\gamma_{1} & =\frac{\theta x_{2}}{\beta_{1} r_{1}(\hat{x})}\left(\alpha+\beta_{1} \frac{E_{1}}{R x_{1}^{2}} r_{1}(\hat{x})+\theta\right), \\
\gamma_{2} & =\frac{\beta_{2} x_{2}}{\beta_{1} r_{1}(\hat{x})} \theta^{2}, \\
\tilde{x}_{i} & =x_{i}-\hat{x}_{i}(i=1, \ldots, 4) .
\end{aligned}
$$

We choose $v_{1}$ and $v_{2}$ such that the system is closed loop stable and achieves a desired setpoint on temperature and concentration.

$$
\begin{aligned}
& v_{1}=-\delta_{1} e_{11}-\delta_{2} e_{12}+\dot{x}_{1}^{r}+v_{11}, \\
& v_{2}=-\delta_{3} e_{21}-\delta_{4} e_{22}+\dot{x}_{3}^{r}+v_{22},
\end{aligned}
$$


where $v_{11}$ and $v_{22}$ are the compensating terms to cope with the error caused by state estimate. $e_{11}=\int\left(x_{1}-x_{1}^{r}\right) d t, e_{12}=x_{1}-x_{1}^{r}, e_{21}=\int\left(x_{3}-x_{3}^{r}\right) d t$, $e_{22}=x_{3}-x_{3}^{r} x_{1}^{r}$ and $x_{3}^{r}$ are respectively the desired reactor temperature and concentration.

The closed-loop error dynamics and observer are given by:

$$
\begin{aligned}
\dot{e}_{11}= & e_{12}, \\
\dot{e}_{12}= & -\delta_{1} e_{11}-\delta_{2} e_{12}+\left(L_{f}\left(x_{1}\right)-\hat{L}_{f}\left(x_{1}\right)\right) \\
& +\left(L_{g_{11}}\left(x_{1}\right)-\hat{L}_{g_{11}}\left(x_{1}\right)\right) u_{1}+v_{11}, \\
\dot{e}_{21}= & e_{22}, \\
\dot{e}_{22}= & -\delta_{3} e_{21}-\delta_{4} e_{22}+\frac{\tilde{x}_{3}}{x_{3}} \dot{y}_{2}-\frac{\tilde{x}_{3}}{x_{3}} L_{f}\left(x_{3}\right)+L_{f}\left(x_{3}\right)-\hat{L}_{f}\left(x_{3}\right)+v_{22}, \\
\dot{\hat{x}}= & f(\hat{x})+g(\hat{x}) u-\left[\frac{\partial \Psi(x)}{\partial x}\right]^{-1} G_{\theta}(C \hat{x}-y) .
\end{aligned}
$$

Now consider the following assumptions:

Assumption 3. The functions $r_{1}(x)$ and $r_{2}(x)$ are globally Lipschitz with respect to $x$, i.e.,

$$
\begin{aligned}
& \forall x \in \mathbb{R}^{4}, \exists \sigma_{r 1}>0,\left\|r_{1}(x)-r_{1}(\hat{x})\right\| \leq \sigma_{r 1}\|\tilde{x}\|, \\
& \forall x \in \mathbb{R}^{4}, \exists \sigma_{r 2}>0,\left\|r_{2}(x)-r_{2}(\hat{x})\right\| \leq \sigma_{r 2}\|\tilde{x}\| .
\end{aligned}
$$

Assumption 4. The control inputs are bounded, i.e.,

$$
\begin{aligned}
& \forall t \geq 0,0<U_{\min 1} \leq u_{1} \leq U_{\max 1}, \\
& \forall t \geq 0,0<U_{\min 2} \leq u_{2} \leq U_{\max 2} .
\end{aligned}
$$

Theorem 2. Consider the controls laws stated in (19)-(20) and the observer (13), if Assumptions 1-4 are satisfied and if the compensating terms $v_{22}$ and $v_{11}$ are chosen as

$$
\begin{gathered}
v_{11}=(\theta-\alpha) \tilde{x}_{1}+\frac{1}{V} \tilde{x}_{3} U_{\min 1}, \\
v_{22}=\left(r_{2}(x)-r_{2}(\hat{x})\right)+\gamma_{1} \tilde{x}_{1}+\gamma_{2} \tilde{x}_{4}
\end{gathered}
$$

and by selecting $\delta_{1}, \delta_{2}, \delta_{3}$ and $\delta_{4}$ such that all roots of the two polynomial $s^{2}+\delta_{2} s+\delta_{1}$ and $s^{2}+\delta_{4} s+\delta_{3}$ lie in the open left-hand side of the complex plane then the closed loop system described by (23) is globally asymptotically stable

Proof. An original proof of Theorem 2 will be presented in the appendix B. 


\section{Simulation results}

Simulations, using MATLAB Software Package, have been carried out to verify the effectiveness of the proposed method.

The values of the model parameters used in simulation are given in Table 1. The states initial conditions were set to:

$$
x(0)=[309.46 ; 4.49 ; 20.06 ; 0]^{T}, \hat{x}(0)=[302.16 ; 2.49 ; 21.06 ; 0.3]^{T} .
$$

First we investigate the transient response of the system for regulation control. For this case the desired temperature and concentration are $350 \mathrm{~K}$ and $20 \mathrm{~mol} / \mathrm{m}^{3}$ respectively.

The temperature and concentration transient responses and the tow controls actions are shown in Figures 2 and 3 respectively.
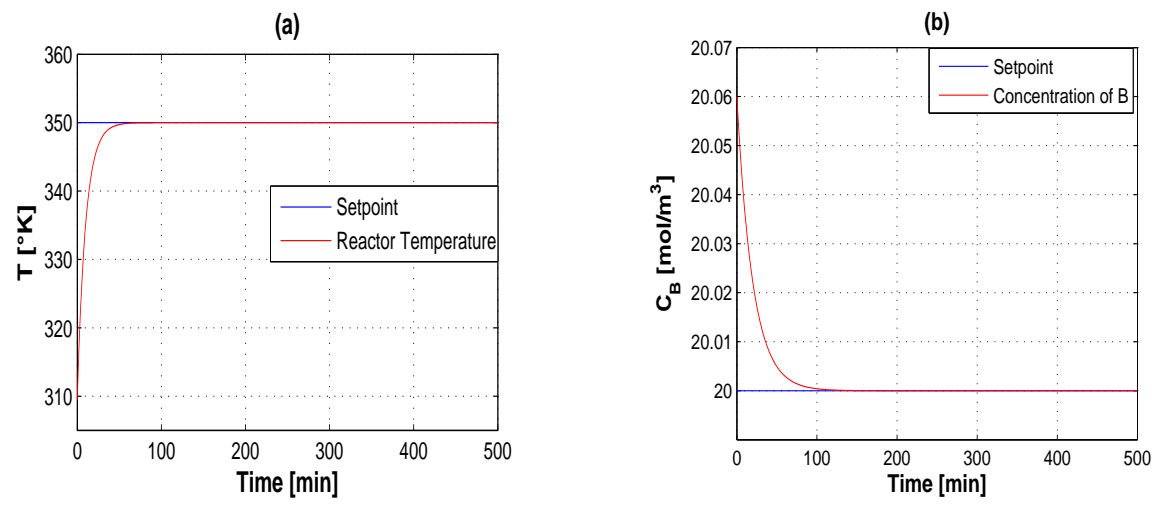

Figure 2. Time response for the: a) Reactor temperature, b) Concentration of the constituent $B$.
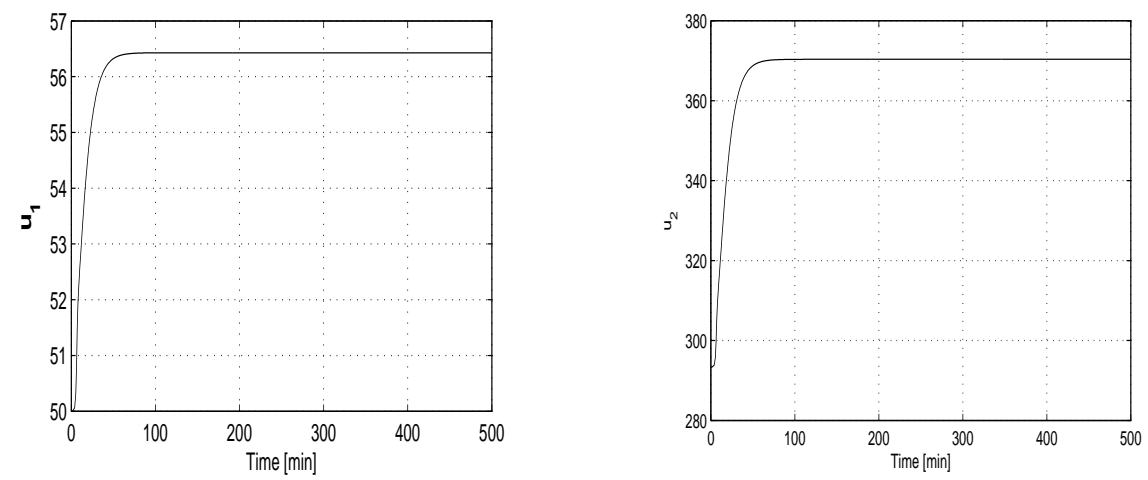

FiguRE 3. Control inputs. 
Next, the proposed controller is tested for the set point tracking. Figure 4 show the measured temperature and concentration and their references trajectories. The manipulated signals $u_{1}$ and $u_{2}$ is also depicted in Figure 5. From the results, it can be seen that the proposed observer/controller structure shows good performance in achieving the output regulation.

Finally, we examine the robustness of the proposed controllers in the presence of the measurements noisy and model uncertainty.

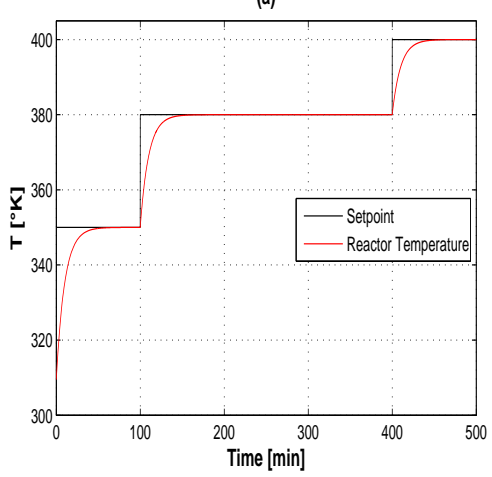

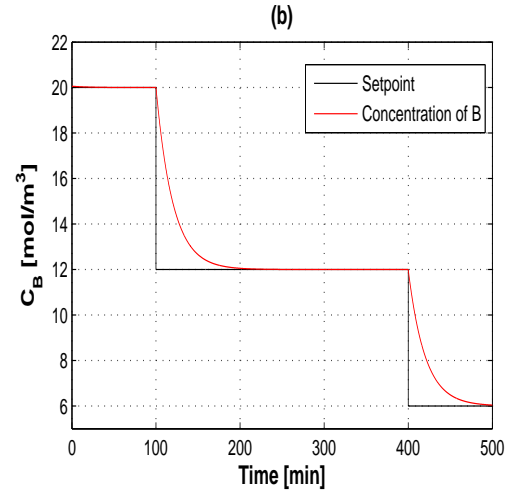

FiguRE 4. Time tracking responses for the: a) Reactor temperature, b) Concentration of the constituent $B$.
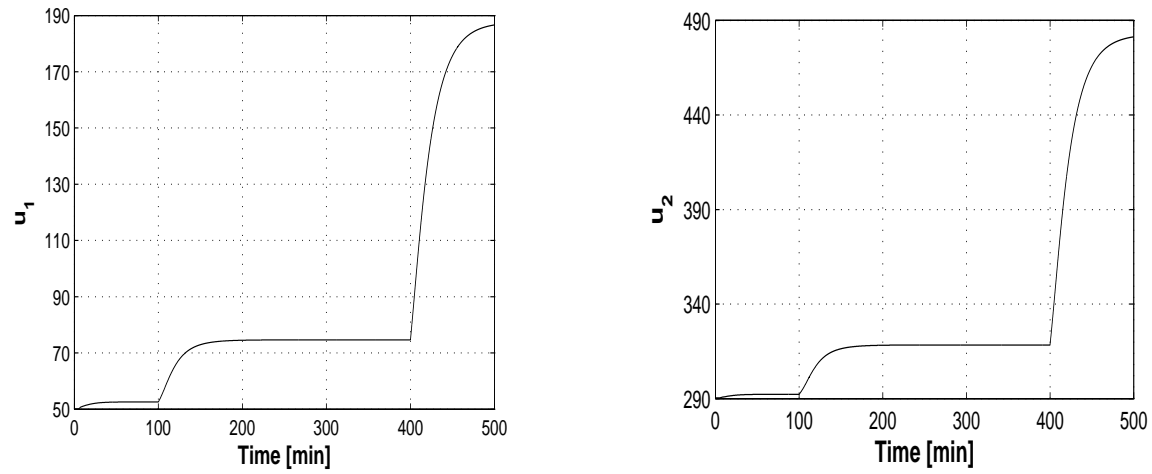

Figure 5. Control inputs for tracking responses.

Case 01: Performance analysis in the presence of measurements noise:

In this case, white Gaussian noises with variances of $5 /$ are simultaneously 
added to the outputs measurements. The temperature and concentration transient's responses for the controller are shown in Figure 6. The tow controls actions obtained in this case are depicted in Figure 7 . It can be seen that the set-point tracking behaviour is very satisfactory. Note that the proposed controller maintains the temperature and concentration in a small neighborhood of the references value despite the noise on the measurement.

(a)

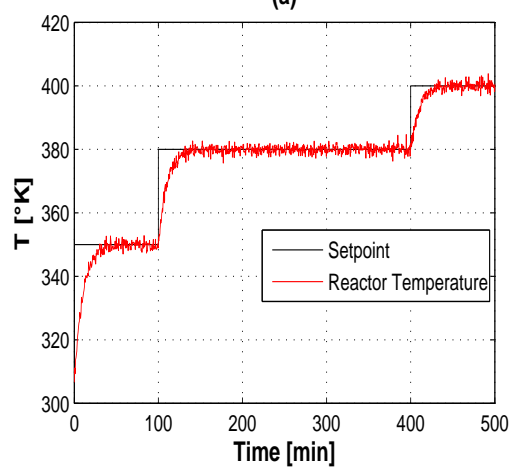

(b)

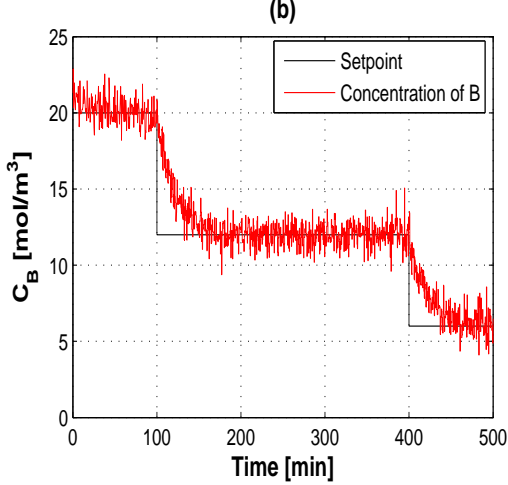

FiguRE 6. Time responses in presence of measure noises for the: a) Reactor temperature, b) Concentration of the constituent $B$.
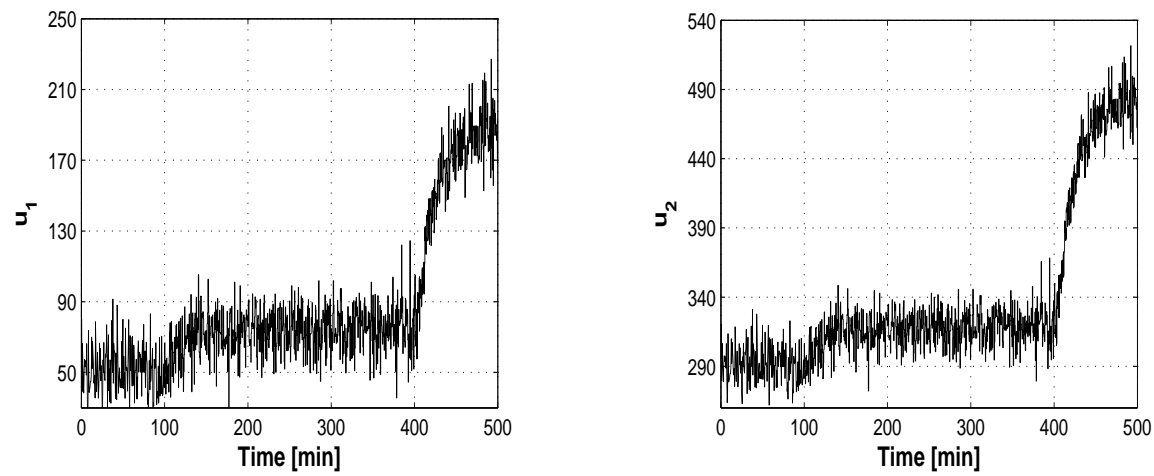

FIGURE 7. Control inputs in presence of measure noises.

Case 02: Performance analysis in the presence of model uncertainty: For this purpose, it was considered a mismatch between the real activation energy and its value in the model. In this work, a difference up to 2 / between the real parameter and its value in the model was considered. Simulation results 
are depicted in Figures 8 and 9. Again the proposed methodology is able to reject the dynamics mismatch adequately.

(a)

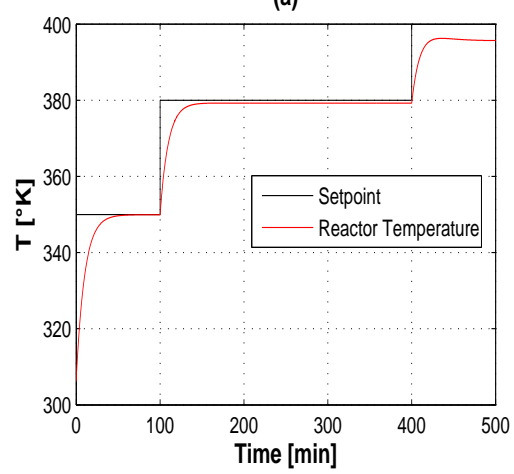

(b)

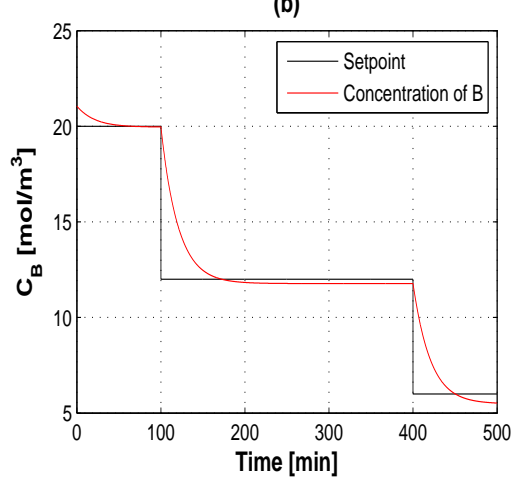

Figure 8 . Time responses for $2 /$ increase in the activation energy for the: a) Reactor temperature, b) Concentration of the constituent $B$.
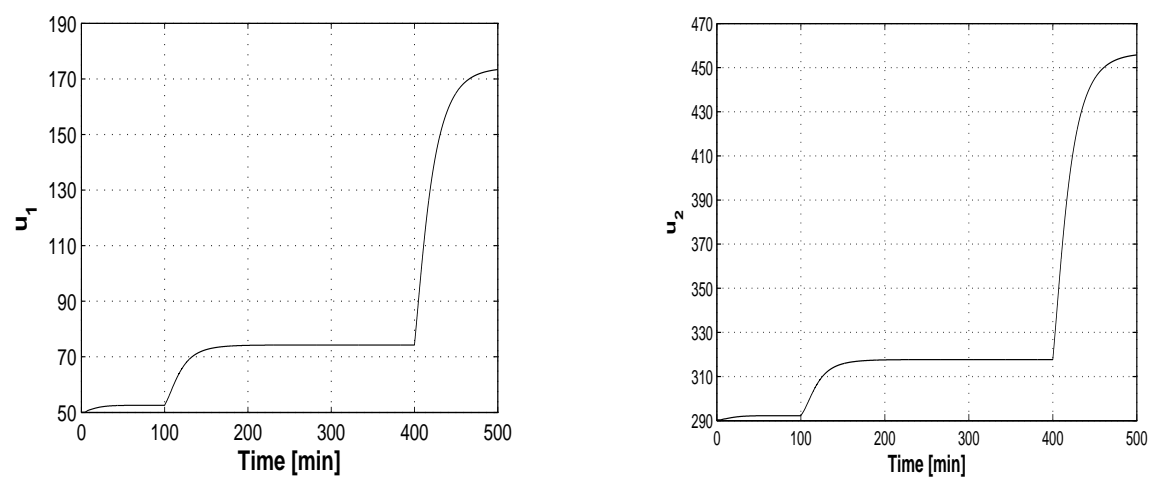

Figure 9. Control input for $2 /$ increase in the activation energy.

\section{Conclusion}

In this paper, we presented a robust observer based on a nonlinear control scheme for a multivariable chemical reactor. The observer proposed in this study offers the advantage of only one tuning parameter $\theta$. This observer is coupled with nonlinear controller. The controller is constructed through feedback linearization for concentration and temperature reactor control. The Lyapunov's stability technique is used to establish the asymptotical stability of 
closed-loop system including the observer dynamics. Through numerical simulations, we illustrated the feasibility of the designed control system. Moreover, the proposed control exhibits a satisfactory performance when used with noisy measurements and dynamics uncertainty.

\section{Appendix}

\section{Appendix A. Proof of Theorem 1}

Let $\tilde{z}=z-\hat{z}$. Then we have:

$$
\dot{\tilde{z}}=\left(A-G_{\theta} C_{z}\right) \tilde{z}+\left[\begin{array}{c}
0 \\
\varphi(z)-\varphi(\hat{Z})
\end{array}\right]+\left[\begin{array}{c}
B_{1}(z, u)-B_{1}(\hat{z}, u) \\
B_{2}(z, u)-B_{2}(\hat{z}, u)
\end{array}\right] .
$$

Consider the following variable change: $\bar{z}=\Delta_{\theta} \tilde{z}$ with $\Delta_{\theta}=\left[\begin{array}{cc}\frac{1}{\theta} & 0 \\ 0 & \frac{1}{\theta^{2}}\end{array}\right]$. Using the well known property of K. Busawon et al. [8]: $\Delta_{\theta} A \Delta_{\theta}^{-1}=\theta A, C_{z} \Delta_{\theta}^{-1}=\theta C_{z}$. We obtain:

$$
\dot{\bar{z}}=\theta\left(A-G_{\theta} C_{z}\right) \bar{z}+\left[\begin{array}{c}
0 \\
\frac{(\varphi(z)-\varphi(\hat{z}))}{\theta^{2}}
\end{array}\right]+\left[\begin{array}{c}
\frac{\left(B_{1}(z, u)-B_{1}(\hat{z}, u)\right)}{\theta} \\
\frac{\left(B_{2}(z, u)-B_{2}(\hat{z}, u)\right)}{\theta^{2}}
\end{array}\right] .
$$

Since $\left(A-G C_{z}\right)$ is Hurwitz, there exists a symmetric positive definite matrix $P$ such that:

$$
\left(A-G C_{z}\right)^{T} P+P\left(A-G C_{z}\right)=-I .
$$

Define $V_{o}(\bar{z})=\bar{z}^{T} P \bar{z}$ as the Lyapunov candidate function. Then its time derivative is:

$$
\dot{V}_{o}(\bar{z})=\dot{\bar{z}}^{T} P \bar{z}+\bar{z}^{T} P \dot{\bar{z}},
$$

$$
\begin{aligned}
\dot{V}_{o}(\bar{z})= & \theta \bar{z}^{T}\left[\left(A-G C_{z}\right)^{T} P+P\left(A-G C_{z}\right)\right] \bar{z} \\
& +2 P \bar{z}^{T}\left[\begin{array}{c}
0 \\
\frac{(\varphi(z)-\varphi(\hat{z}))}{\theta^{2}}
\end{array}\right]+2 P \bar{z}^{T}\left[\begin{array}{c}
\frac{\left(B_{1}(z, u)-B_{1}(\hat{z}, u)\right)}{\theta} \\
\frac{\left(B_{2}(z, u)-B_{2}(\hat{z}, u)\right)}{\theta^{2}}
\end{array}\right],
\end{aligned}
$$

$$
\dot{V}_{o}(\bar{z})=-\theta\|\bar{z}\|^{2}+2 P \bar{z}^{T}\left[\begin{array}{c}
0 \\
\frac{(\varphi(z)-\varphi(\hat{z}))}{\theta^{2}}
\end{array}\right]+2 P \bar{z}^{T}\left[\begin{array}{c}
\frac{\left(B_{1}(z, u)-B_{1}(\hat{z}, u)\right)}{\theta} \\
\frac{\left(B_{2}(z, u)-B_{2}(\hat{z}, u)\right)}{\theta^{2}}
\end{array}\right] .
$$

Setting:

$$
\Delta_{\theta_{0}}=\left[\begin{array}{cc}
\frac{1}{\theta_{0}} & 0 \\
0 & \frac{1}{\theta_{0}^{2}}
\end{array}\right]
$$


By introducing $\Delta_{\theta_{0}}$ in $(29)$, we obtain:

$$
\begin{gathered}
\dot{V}_{o}(\bar{z})=-\theta\|\bar{z}\|^{2}+2 \Delta_{\theta_{0}} P \bar{z}^{T}\left[\begin{array}{c}
0 \\
\frac{\theta_{0}^{2}}{\theta^{2}}(\varphi(z)-\varphi(\hat{z}))
\end{array}\right] \\
+2 \Delta_{\theta_{0}} P \bar{z}^{T}\left[\begin{array}{c}
\frac{\theta_{0}}{\theta^{2}}\left(B_{1}(z, u)-B_{1}(\hat{z}, u)\right) \\
\frac{\theta_{0}^{2}}{\theta^{2}}\left(B_{2}(z, u)-B_{2}(\hat{z}, u)\right)
\end{array}\right], \\
\dot{V}_{o}(\bar{z}) \leq-\theta\|\bar{z}\|^{2}+2\left\|\Delta_{\theta_{0}}\right\|\left\|P \bar{z}^{T}\right\|\left\langle\left[\begin{array}{c}
0 \\
\frac{\theta_{0}^{2}}{\theta^{2}}(\varphi(z)-\varphi(\hat{z}))
\end{array}\right]\right\rangle \\
+2\left\|\Delta_{\theta_{0}}\right\|\left\|P \bar{z}^{T}\right\|\left\langle\left[\begin{array}{c}
\left.\left.\frac{\theta_{0}}{\theta}\left(B_{1}(z, u)-B_{1}(\hat{z}, u)\right)\right]\right\rangle \\
\frac{\theta_{0}^{2}}{\theta^{2}}\left(B_{2}(z, u)-B_{2}(\hat{z}, u)\right)
\end{array}\right]\right\rangle,
\end{gathered}
$$

where $\langle\cdot\rangle$ is the Euclidean scalar product on $\mathbb{R}^{n}$. Using the Cauchy-Schwarz inequality this gives for $\theta>\theta_{0}$ sufficiently large:

$$
\begin{aligned}
\dot{V}_{o}(\bar{z}) \leq & -\theta\|\bar{z}\|^{2}+2\left\|\Delta_{\theta_{0}}\right\|\left\|P \bar{z}^{T}\right\| \frac{\theta_{0}^{2}}{\theta^{2}}\|(\varphi(z)-\varphi(\hat{z}))\|+2\left\|\Delta_{\theta_{0}}\right\|\left\|P \bar{z}^{T}\right\| \\
& \times \sqrt{\left(\frac{\theta_{0}}{\theta}\right)^{2}\left\|B_{1}(z, u)-B_{1}(\hat{z}, u)\right\|^{2}+\left(\frac{\theta_{0}^{2}}{\theta^{2}}\right)^{2}\left\|B_{2}(z, u)-B_{2}(\hat{z}, u)\right\| .}
\end{aligned}
$$

Using the following assumption (Assumptions 1 and 2):

$$
\begin{aligned}
\|(\varphi(z)-\varphi(\hat{z}))\| & \leq \lambda_{\varphi}\|\tilde{z}\| \\
& \Rightarrow\|(\varphi(z)-\varphi(\hat{z}))\| \leq \lambda_{\varphi} \sqrt{\theta^{2}\left\|\bar{z}_{1}\right\|^{2}+\theta^{4}\left\|\bar{z}_{2}\right\|^{2}}, \\
\left\|B_{1}(z, u)-B_{1}(\hat{z}, u)\right\| & \leq \lambda_{B_{1}}\|\tilde{z}\| \\
& \Rightarrow\left\|B_{1}(z, u)-B_{1}(\hat{z}, u)\right\| \leq \lambda_{B_{1}} \sqrt{\theta^{2}\left\|\bar{z}_{1}\right\|^{2}+\theta^{4}\left\|\bar{z}_{2}\right\|^{2}} \\
\left\|B_{2}(z, u)-B_{2}(\hat{z}, u)\right\| & \leq \lambda_{B_{2}}\|\tilde{z}\| \\
& \Rightarrow\left\|B_{2}(z, u)-B_{2}(\hat{z}, u)\right\| \leq \lambda_{B_{2}} \sqrt{\theta^{2}\left\|\bar{z}_{1}\right\|^{2}+\theta^{4}\left\|\bar{z}_{2}\right\|^{2}},
\end{aligned}
$$

where $\lambda_{\varphi}, \lambda_{B_{1}}$ and $\lambda_{B_{2}}$ denote, respectively, the Lipschitz constants of $\varphi(z)$, $B_{1}(z, u)$ and $B_{2}(z, u)$.

Inequality (32) becomes:

$$
\begin{aligned}
\dot{V}_{o}(\bar{z}) \leq & -\theta\|\bar{z}\|^{2}+2 \lambda_{\varphi} \frac{\theta_{0}^{2}}{\theta_{M}}\left\|\Delta_{\theta_{0}}\right\|\left\|P \bar{z}^{T}\right\| \sqrt{\left\|\overline{z_{1}}\right\|^{2}+\theta^{2}\left\|\bar{z}_{2}\right\|^{2}}+2\left\|\Delta_{\theta_{0}}\right\|\left\|P \bar{z}^{T}\right\| \\
& \times \sqrt{\left(\lambda_{B_{1}}^{2} \theta_{0}^{2}+\lambda_{B_{2}}^{2} \theta_{0}^{4}\right)\left(\left\|\bar{z}_{1}\right\|^{2}+\left\|\bar{z}_{2}\right\|^{2}\right)}
\end{aligned}
$$

$$
\dot{V}_{o}(\bar{z}) \leq-\theta\|\bar{z}\|^{2}+2 \theta_{0} \lambda_{\varphi}\left\|P \bar{z}^{T}\right\|\|\bar{z}\|+2 \theta_{0} \lambda_{B} \mid\left\|P \bar{z}^{T}\right\|\|\bar{z}\|,
$$


where $\gamma_{B}=\sqrt{\lambda_{B_{1}}^{2}+\lambda_{B_{2}}^{2} \theta_{0}^{2}}$.

Thus, it results that:

$$
\begin{aligned}
& \dot{V}_{o}(\bar{z}) \leq-\theta\|\bar{z}\|^{2}+\xi\|\bar{z}\|^{2}, \\
& \dot{V}_{o}(\bar{z}) \leq-(\theta-\xi)\|\bar{z}\|^{2},
\end{aligned}
$$

where $\xi=2 \theta_{0}\left(\lambda_{\varphi}+\lambda_{B}\right) \lambda_{\max }^{P}$ and $\lambda_{\max }^{P}$ denotes the largest eigenvalue of $P$.

Then, if $\theta>\xi$ the estimation error converges exponentially to zero.

The system (8) is considered to be uniformly observable. Then:

$$
z=\Psi(x) \text { and } x=\Psi^{-1}(z) .
$$

If the transform $\Psi(x)$ is nonsingular and $\Psi^{-1}(z)$ is uniformly Lipschitz, we have:

$$
\|x-\hat{x}\| \leq \bar{\mu}_{\Psi}\|z-\hat{z}\|
$$

where $\bar{\mu}_{\Psi}$ denote, the Lipschitz constants of $\Psi^{-1}(z)$.

Thus, the inequality (36) can be rewritten in the original coordinates as:

$$
\dot{V}_{o}(\tilde{x}) \leq-\bar{\mu}_{\Psi}(\theta-\xi)\|\tilde{x}\|^{2} .
$$

If we chose $\theta>\xi$, then the norm of the estimation error goes to zero as $t \rightarrow \infty$. Consequently, the convergence of the algorithm is guaranteed. This completes the proof of the theorem.

\section{Appendix B. Proof of Theorem 2}

Consider the following Lyapunov function candidate:

$$
V\left(\tilde{x}, e_{1}, e_{2}\right)=V_{o}(\tilde{x})+V_{c 1}\left(e_{1}\right)+V_{c 2}\left(e_{2}\right),
$$

where $V_{c 1}\left(e_{1}\right)=\frac{1}{2}\left(e_{11}^{2}+e_{12}^{2}\right), V_{c 2}\left(e_{2}\right)=\frac{1}{2}\left(e_{21}^{2}+e_{22}^{2}\right)$.

The time derivative of the Lyapunov $V\left(\tilde{x}, e_{1}, e_{2}\right)$ function is:

$$
\dot{V}\left(\tilde{x}, e_{1}, e_{2}\right)=\dot{V}_{o}(\tilde{x})+\dot{V}_{c 1}\left(e_{1}\right)+\dot{V}_{c 2}\left(e_{2}\right) .
$$

Where $\dot{V}_{o}(\tilde{x})$ was defines in the preceding proof $\left(\dot{V}_{o}(\tilde{x}) \leq-\bar{\mu}_{\Psi}(\theta-\xi)\|\tilde{x}\|^{2}\right)$ :

$$
\dot{V}_{c 1}\left(e_{1}\right)=e_{11} \dot{e}_{11}+e_{12} \dot{e}_{12},
$$

$$
\begin{aligned}
\dot{V}_{c 1}\left(e_{1}\right)= & e_{11} e_{12}+e_{12}\left(-\delta_{1} e_{11}-\delta_{2} e_{12}\right)+e_{12}\left((\alpha-\theta) \tilde{x}_{1}+\beta_{1}\left(r_{1}(x)-r_{1}(\hat{x})\right)\right) \\
& +e_{12} \beta_{2}\left(r_{2}(x)-r_{2}(\hat{x})\right)-\frac{1}{V} \tilde{x}_{3} u_{1} e_{12}+e_{12} v_{11}
\end{aligned}
$$

$$
\begin{aligned}
\dot{V}_{c 1}\left(e_{1}\right)= & -e_{1}^{T} A_{c 1} e_{1}+e_{12}\left((\alpha-\theta) \tilde{x}_{1}+\beta_{1}\left(r_{1}(x)-r_{2}(\hat{x})\right)\right) \\
& +e_{12} \beta_{2}\left(r_{2}(x)-r_{2}(\hat{x})\right)-\frac{1}{V} \tilde{x}_{3} u_{1} e_{12}+e_{12} v_{11}
\end{aligned}
$$

with $A_{c 1}=\left[\begin{array}{cc}0 & -1 \\ \delta_{1} & \delta_{2}\end{array}\right]>0, e_{1}=\left[\begin{array}{l}e_{11} \\ e_{12}\end{array}\right]$. 
Using assumption (Assumption 3), inequality (43) become

$$
\begin{aligned}
\dot{V}_{c 1}\left(e_{1}\right) \leq & -\lambda_{A_{c 1}}^{\min }\left\|e_{1}\right\|^{2}+e_{12}(\alpha-\theta) \tilde{x}_{1}+\beta_{1} \sigma_{r 1} e_{12}\|\tilde{x}\| \\
& +\beta_{2} \sigma_{r 2} e_{12}\|\tilde{x}\|-\frac{1}{V} \tilde{x}_{3} U_{\min 1} e_{12}+e_{12} v_{11},
\end{aligned}
$$

where $\lambda_{A_{c 1}}^{\min }$ is the minimum eigenvalue of $A_{c 1}$.

Knowing that $\left\|e_{12}\right\| \leq\left\|e_{1}\right\|,\left\|\tilde{x}_{3}\right\| \leq\|\tilde{x}\|$ and $\left\|\tilde{x}_{1}\right\| \leq\|\tilde{x}\|$ we obtain:

$$
\begin{aligned}
\dot{V}_{c 1}\left(e_{1}\right) \leq & -\lambda_{A_{c 1}}^{\min }\left\|e_{1}\right\|^{2}+\beta_{1} \sigma_{r 1}\left\|e_{1}\right\|\|\tilde{x}\|+\beta_{2} \sigma_{r 2}\left\|e_{1}\right\|\|\tilde{x}\| \\
& +(\alpha-\theta) \tilde{x}_{1} e_{12}-\frac{1}{V} \tilde{x}_{3} U_{\min 1} e_{12}+e_{12} v_{11} .
\end{aligned}
$$

If we choose $v_{11}$ as,

$$
v_{11}=(\theta-\alpha) \tilde{x}_{1}+\frac{1}{V} \tilde{x}_{3} U_{\min 1}
$$

inequality (45) becomes:

$$
\begin{aligned}
& \dot{V}_{c 1}\left(e_{1}\right) \leq-\lambda_{A_{c 1}}^{\min }\left\|e_{1}\right\|^{2}+\left(\beta_{1} \sigma_{r 1}+\beta_{2} \sigma_{r 2}\right)\left\|e_{1}\right\|\|\tilde{x}\|, \\
& \text { (48) } \dot{V}_{c 2}\left(e_{2}\right)=e_{21} \dot{e}_{21}+e_{22} \dot{e}_{22} \text {, } \\
& \dot{V}_{c 2}\left(e_{2}\right)=e_{21} e_{22}+e_{22}\left(-\delta_{3} e_{21}-\delta_{4} e_{22}\right)+e_{22}\left(\left(r_{1}(x)-r_{1}(\hat{x})\right)\right) \\
& -e_{22}\left(\left(r_{2}(x)-r_{2}(\hat{x})\right)+\gamma_{1} \tilde{x}_{1}+\gamma_{2} \tilde{x}_{4}-\frac{1}{V} \tilde{x}_{3} u_{1}\right)+e_{22} v_{22} \text {, } \\
& \dot{V}_{c 2}\left(e_{2}\right)=-e_{2}^{T} A_{c 2} e_{2}+e_{22}\left(\left(r_{1}(x)-r_{1}(\hat{x})\right)\right) \\
& -e_{22}\left(\left(r_{2}(x)-r_{2}(\hat{x})\right)+\gamma_{1} \tilde{x}_{1}+\gamma_{2} \tilde{x}_{4}-\frac{1}{V} \tilde{x}_{3} u_{1}\right)+e_{22} v_{22},
\end{aligned}
$$

with $A_{c 2}=\left[\begin{array}{cc}0 & -1 \\ \delta_{3} & \delta_{4}\end{array}\right]>0, e_{2}=\left[\begin{array}{l}e_{21} \\ e_{22}\end{array}\right]$, where $\lambda_{A_{c 2}}^{\min }$ is the minimum eigenvalue of $A_{c 2}$.

$$
\begin{aligned}
\dot{V}_{c 2}\left(e_{2}\right) \leq & -\lambda_{A_{c 2}}^{\min }\left\|e_{2}\right\|^{2}+\sigma_{r 2}\|\tilde{x}\| e_{22} \\
& -e_{22}\left(\left(r_{2}(x)-r_{2}(\hat{x})\right)+\gamma_{1} \tilde{x}_{1}+\gamma_{2} \tilde{x}_{4}-\frac{1}{V} \tilde{x}_{3} u_{1}\right)+e_{22} v_{22} .
\end{aligned}
$$

We have: $\left\|e_{22}\right\| \leq\left\|e_{2}\right\|$ and $\left\|\tilde{x}_{3}\right\| \leq\|\tilde{x}\|$.

Then:

$$
\begin{aligned}
\dot{V}_{c 2}\left(e_{2}\right) \leq & -\lambda_{A_{c 2}}^{\min }\left\|e_{2}\right\|^{2}+\sigma_{r 2}\|\tilde{x}\|\left\|e_{2}\right\| \\
& -e_{22}\left(\left(r_{2}(x)-r_{2}(\hat{x})\right)+\gamma_{1} \tilde{x}_{1}+\gamma_{2} \tilde{x}_{4}+\frac{1}{V} U_{\min 1}\right)+e_{22} v_{22} .
\end{aligned}
$$

We choose $v_{22}=\left(r_{2}(x)-r_{2}(\hat{x})\right)+\gamma_{1} \tilde{x}_{1}+\gamma_{2} \tilde{x}_{4}+\frac{1}{V} U_{\min 1}$, inequality (52) becomes:

$$
\begin{gathered}
\dot{V}_{c 2}\left(e_{2}\right) \leq-\lambda_{A_{c 2}}^{\min }\left\|e_{2}\right\|^{2}+\sigma_{r 2}\|\tilde{x}\|\left\|e_{2}\right\| \\
\dot{V}\left(\tilde{x}, e_{1}, e_{2}\right) \leq \\
-\mu_{\Psi}(\theta-\xi)\|\tilde{x}\|^{2}-\lambda_{A_{c 1}}^{\min }\left\|e_{1}\right\|^{2}-\lambda_{A_{c 2}}^{\min }\left\|e_{2}\right\|^{2} \\
+\left(\beta_{1} \sigma_{r 1}+\beta_{2} \sigma_{r 2}\right)\left\|e_{1}\right\|\|\tilde{x}\|+\sigma_{r 2}\|\tilde{x}\|\left\|e_{2}\right\| .
\end{gathered}
$$


Inequality (54) can be rewritten as:

$$
\dot{V}\left(\tilde{x}, e_{1}, e_{2}\right) \leq-\Lambda^{T} \Gamma \Lambda
$$

with

$$
\Gamma=\left[\begin{array}{ccc}
\mu_{\Psi}(\theta-\xi) & -\left(\beta_{1} \sigma_{r 1}+\beta_{2} \sigma_{r 2}\right) & -\sigma_{r 2} \\
0 & \lambda_{A_{c 1}}^{\min } & 0 \\
0 & 0 & \lambda_{A_{c 2}}^{\min }
\end{array}\right], \Lambda=\left[\begin{array}{c}
\tilde{x} \\
e_{1} \\
e_{2}
\end{array}\right]
$$

If $\theta>\xi, \lambda_{A_{c 1}}^{\min }>0$ and $\lambda_{A_{c 2}}^{\min }>0$, then $\Gamma>0$ and $V\left(\tilde{x}, e_{1}, e_{2}\right) \leq 0$.

Consequently, asymptotical stability of the closed-loop system is established.

\section{References}

[1] R. Aguilar, Sliding-mode observer for uncertainty estimation in a class of chemical reactor: A differential-algebraic approach, Chemical Product and Process Modeling 2 (2007), no. 3, p. 10.

[2] R. Aguilar and R. Martinez-Guerra, State estimation for nonlinear systems under model unobservable uncertainties: application to continuous reactor, Chemical Engineering Journal 108 (2005), no. 1-2, 139-144.

[3] R. Aguilar, R. Martinez-Guerra, and R. Maya-Yescas, State estimation for partially unknown nonlinear systems: a class of integral high gain observers, IEE Proc. Contr. Theor. Appl. 150 (2003), no. 3, 240-244.

[4] R. Aguilar, R. Martinez-Guerra, and A. Poznyak, Reaction heat estimation in continuous chemical reactors using high gain observers, Chemical Engineering Journal $\mathbf{8 7}$ (2002), 351-356.

[5] T. Ahmed-Ali and F. Lamnabhi-Lagarrigue, Sliding observer-controller design for uncertain triangular nonlinear systems, IEEE Trans. Automat. Control 44 (1999), no. 6, 1244-1249.

[6] C. Canudas de Wit and J. Slotine, Sliding observers for robot manipulators, Automatica J. IFAC 27 (1991), no. 5, 859-864.

[7] B. Daaou, A. Mansouri, M. Bouhamida, and M. Chenafa, A robust nonlinear observer for state variables estimation in multi-input multi-output chemical reactors, International Journal of Chemical Reactor Engineering 6 (2008), A86.

[8] M. Farza, K. Busawon, and H. Hammouri, Simple nonlinear observers for on-line estimation of kinetic rates in bioreactors, Automatica J. IFAC 34 (1998), no. 3, 301-318.

[9] M. Farza, H. Hammouri, C. Jallut, and J. Lieto, State observation of a nonlinear system: application to (bio)chemical processes, A. I. Ch. E. Journal 45 (1999), no. 1, 93-106.

[10] J. P. Gauthier and G. Bonard, Observability for any $u(t)$ of a class of nonlinear systems, IEEE Trans. Automat. Control 26 (1981), no. 4, 922-926.

[11] J. P. Gauthier, H. Hammouri, and S. A. Othman, A simple observer for nonlinear systems applications to bioreactors, IEEE Trans. Automat. Control 37 (1992), no. 6, 875-880.

[12] J. Gonzalez-Trejo, J. A. Ramirez, and G. Fernandez, Robust control with uncertainty estimation for feedback linearizable systems: application to control of distillation columns, J. Proc. Cont. 9 (1999), no. 3, 221-231.

[13] M. Groebel, F. Allgöwer, M. Storz, and E. D. Gilles, Asymptotically exact I/Olinearization of an industrial distillation column, Proceedings of the 1995 American Control Conference (ACC 95), Seattle, Washington, USA, 21-23, pp. 2648-2652, June 1995.

[14] M. Henson and D. Seborg, Input/output linearization of general nonlinear processes, AIChE Journal 36 (1990), no. 11, 1753-1757. 
[15] A. Isidori, Nonlinear Control Systems, Springer-Verlag, New York, 1989.

[16] A. K. Jana, A. N. Samanta, and S. Ganguly, Globally linearized control on diabatic continuous stirred tank reactor: a case study, ISA Trans. 44 (2005), no. 3, 423-444.

[17] H. K. Khalil, Nonlinear Systems, second ed. Prentice-Hall, Upper Saddle River, NJ, 1996.

[18] N. Kravaris and C. Chung, Nonlinear state feedback synthesis by global input/output linearization, AIChE J. 33 (1987), no. 4, 592-603.

[19] J. D. Morningred, B. E. Paden, D. E. Seborg, and D. A. Mellichamp, An adaptive nonlinear predictive controller, Proc. Am. Control Conf. 2 (1990), 1614-1619; Chemical Engineering Science 47 (1992), no. 4, 755-762.

[20] M. I. Natarajan and E. F. Andrew, Design of a stable adaptive nonlinear observer for an exothermic stirred tank reactor, Computers chem. Engng 20 (1996), no. 9, 1141-1147.

[21] S. S. Sastry and A. Isidori, Adaptive control of linearizable systems, IEEE Trans. Automat. Control 34 (1989), no. 11, 1123-1131.

[22] J. Slotine, J. K. Hedrick, and E. A. Misawa, On sliding observers for nonlinear systems, ASME Journal of Dynamics and System Measurement Control 109 (1987), no. 3, 245252.

[23] E. Slotine and W. Li, Applied Nonlinear Control, Prentice-Hall, New Jersey, 1991.

[24] J.-J. E. Slotine and J. K. Hedrick, Robust input-output feedback linearization, Internat. J. Control 57 (1993), no. 5, 1133-1139.

[25] D. Tyner, M. Soroush, and C. G. Grady, Adaptive temperature control of multiproduct jacketed reactors, Industrial and Engineering Chemistry Research 38 (1999), no. 11, 4337-4344.

[26] G. Wang, S. Peng, and H. Huang, A sliding observer for nonlinear process control, Chemical Engineering Science 52 (1997), no. 5, 787-805.

BOUHAMIdA MOHAMED

Department of Automation

FACUlty of Electrical Engineering

University OF SCIENCE AND TEChNOLOGY

B.P 1505 El Mn'Aouer, Oran, Algeria

E-mail address: m_bouhamida@yahoo.com

DAAOU BACHIR

Department of Sciences and Technology

IBN-KHALDOUN UNIVERSITY

BP 78, ZaAroura Road, Tiaret, Algeria

AND

Department of Electrical Engineering

E. N. S. E. T

Laboratory of Automatics and Systems Analysis (L.A.A.S)

BP 1523, El' M'NAOUer, Oran, Algeria

E-mail address: daaou_b@yahoo.fr

Mansouri AbDellah

Department of Electrical EngineERing

E. N. S. E. T

Laboratory of Automatics and Systems Analysis (L.A.A.S)

BP 1523, El' M'NaOuer, Oran, Algeria

E-mail address: mansouri@enset-oran.dz 
Chenafa Mohammed

Department of Electrical Engineering

E. N. S. E. T

Laboratory of Automatics and Systems Analysis (L.A.A.S)

BP 1523, El' M'Naouer, Oran, Algeria

E-mail address: chenafa@enset-oran.dz 